\title{
A Design of Snoring Detection System using Chaotic Signal
}

\author{
Yeon-Gyu Choo, Member, KIMICS
}

\begin{abstract}
In this study, the existence of chaotic characteristics in snoring signals obtained in the form of time series data was checked through quantitative and qualitative analysis methods, and a snoring signal detection system was designed applied with detection algorithms considering diverse parameters of occurring signals in order to enhance the accuracy and reliability of detections and the performance of the system was checked. The system was tested with certain snoring patients and thereby the results as follows could be obtained.
\end{abstract}

Index Terms-Chaotic signal, Detection, Snoring, Time series signal

\section{INTRODUCTION}

Snoring is noises occurring due to air flows during respiration which is a phenomenon that occurs because negative pressure is formed to pull loose tissues in respiratory tract so that the tissue vibrate when the tension of the muscles of the palate, the tongue or the pharynx, when the tonsil is too large, when the soft palate and the uvula are too long, or when there are anatomical or functional strictures in the nose due to a deformation or splenic tumors. As studies on sleeping have advanced recently, it has been known that severe snoring patients would complain about fatigue after sleeping like the fatigue they would feel if they did not sleep and a lack of comfortable sleep, have higher blood pressure compared to those who do not snore and also have painful crises and angina pectoris more frequently and thus snoring can work as a risk factor for cardiovascular diseases.[10]

In addition, severe long-term snoring is associated with progress into the sleeping disorder that requires awakening to segment sleep to relieve chronic low ventilation of the alveoli, that is, the obstructive sleep apnea syndrome.

Diverse medical techniques to effectively cope with snoring are currently being introduced. In particular, representative examples range from non-surgical therapies such as correcting sleeping postures, reducing body weights,

\footnotetext{
Manuscript received August 20, 2010; revised September 20, 2010; accepted September 25, 2010.

Yeon-Gyu Choo is with the Department of Electronic Engineering, Jinju National University, Jinju, 660-758, Korea (Email: ygchoo@jinju.ac.kr)
}

controlling drinking and drug taking and using breathing apparatuses to surgical therapies to expand narrowed respiratory tracts laser or radio-frequencies. In particular, of the non-surgical therapies, correcting sleeping postures is known to be the most economic and effective method recommended for general snoring patients.

Currently, there are apparatuses to prevent snoring such as positive pressure oxygen respirators, sprayers, electronic stimulators, surgeries and oral assist devices. However, they are too expensive for personal uses and thus they are not suitable to be applied to general snoring patients and they have the risk of adverse events depending on users. Since it is important to check whether snoring occurred as a representative symptom as such, efforts to develop snoring detection algorithms have been steadily continued.

In this paper, it will be checked whether snoring has chaotic characteristics using the time series data of snoring and if snoring has chaotic characteristics, a system to perceive snoring by detecting the periodicity that appears intermittently will be proposed.

So far, in relation to the perception of snoring, studies to detect snoring by detecting frequency characteristics from signals of changes in air pressures in the masks of positive pressure oxygen respirators through FFT and inputting the signals into the neural net, studies to detect snoring using the frequency characteristics obtained from simple FFT, studies to detect sections using distributions and detect snoring using the neural net and studies of algorithms to detect snoring using error back propagation neural nets have been conducted.

In this paper, it will be checked whether snoring has chaotic characteristics using the time series data of snoring and if snoring has chaotic characteristics, a system to perceive snoring by detecting the periodicity that appears intermittently will be proposed.[7][11][12]

\section{ANALYSIS OF CHAOTIC SIGNALS IN SNORING SIGNALS}

\section{2-1. Time series signal}

Time series analysis methods are generally used to forecast changes in the states of systems under the premise that past changes in systems over time will continue in future too. If changes in states have periodicity, accurate forecasting becomes possible through system modeling. Therefore, the passage of time 
becomes the most important variable and the non-periodic and chaotic characteristics of the signals of snoring patients changing over time are analyzed.[1][2][3]

Although time series analysis methods can be appropriately used in cases where there is no rapid change, by the nature of snoring signals, these methods are not accurate if there are variables such as differences between males and females, ages and the existence of diseases that are important causes of snoring. Therefore, time series analysis methods are used to analyze the existence of chaotic signals in snoring signals that have non-linear characteristics stronger than linear characteristics.

The time series data used in analyzing chaotic signals in this paper were snoring voice signals occurring for 6 hours of sleeping obtained from an adult male in his $30 \mathrm{~s}$ through a directional mike with a sampling frequency of $22[\mathrm{kHz}]$ in a mono form. The chaotic characteristics of snoring signals will be analyzed by quantitative and qualitative methods and snoring signals will be detected through detecting intermittent periodicity.

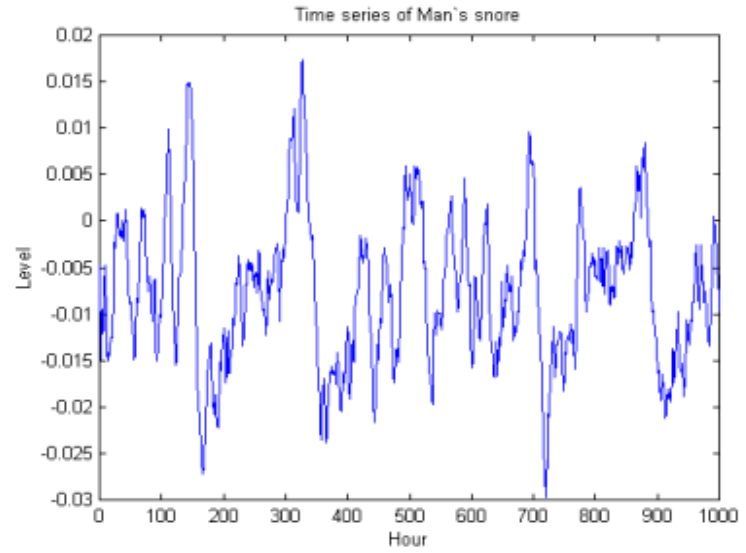

Fig. 1. Time series of man's snore

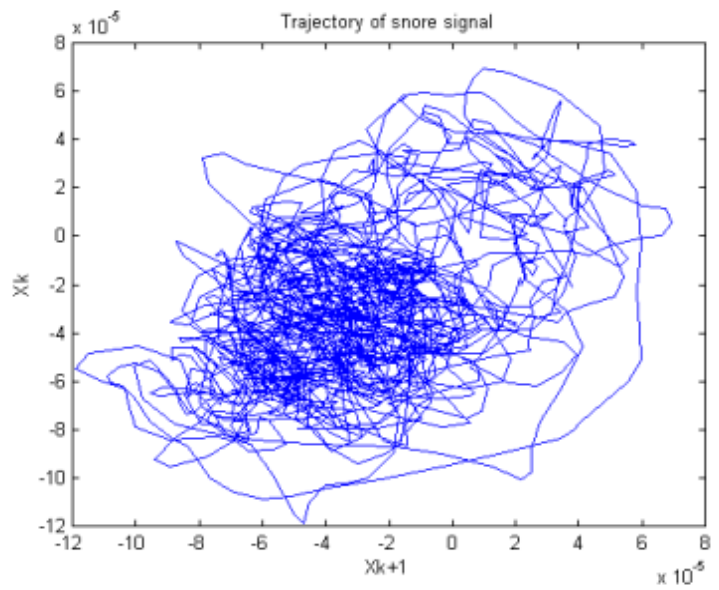

Fig. 2. The attractor of time series data

The time series data shown in Figure 1 show a part of changes in snoring signals over time and in the horizontal axis, changes in signal sizes over time and intermittent periodicity distinguished from noise signals can be observed.[2][6][8]

The attracters of the time series data are expressed in a phase space as shown in Figure 2 and where, the horizontal axis and the vertical axis show state values by time in the phase space considering time delays. On observing the forms of the attracters, they are clearly distinguished from periodic signals and the random state distributions across the phase space observed in noise signals cannot be identified. The existence of chaotic characteristics can become clear through additional quantitative analyses and qualitative analyses.[4][9]

\section{2-2. Quantitative analysis}

The correlation dimension used as a quantitative analysis method in this paper is obtained by reconstructing the time series signals composed of data in the form of one dimensional vectors into an embedding dimension and then using the degrees of interference between traces within the radius while changing the radius within the range.[5] For the precision of the correlation dimension, time series data obtained in the unit of msec were used. Table 1 shows the correlation dimensions obtained while changing the embedding dimension from 1 to 6 and Figure 3 shows the correlation integral used to obtain the correlation dimensions as a graph.

TABLE. 1.

CORRELATION DIMENSION(C.D.) OF TIME SERIES DATA IN TERMS OF EMBEDDING DIMENSION(E.D.)

\begin{tabular}{|c|c|c|c|c|c|c|}
\hline E.D. & 1 & 2 & 3 & 4 & 5 & 6 \\
\hline C.D. & 0.8253 & 1.6950 & 2.3943 & 2.4373 & 2.2468 & 1.8763 \\
\hline
\end{tabular}

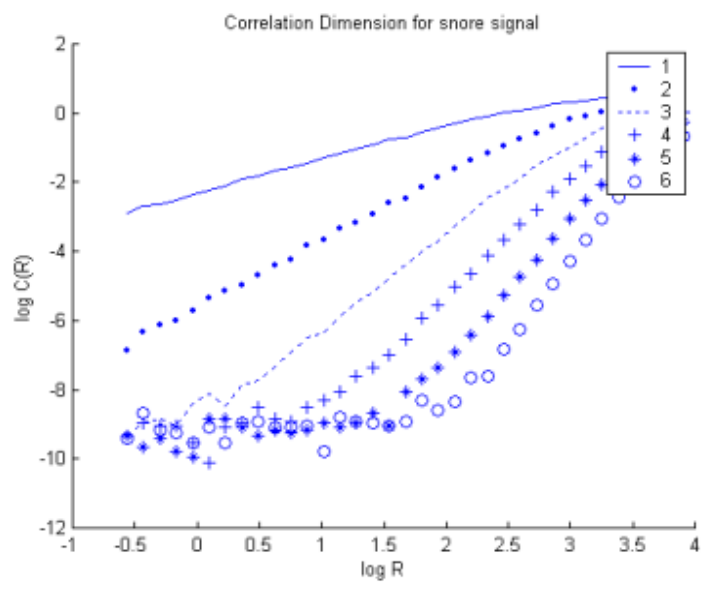

Fig. 3. Correlation integral of time series data

Based on the results of simulations, it could be seen that the radius to reach the normal state of the correlation integral differed greatly depending on embedding dimensions as shown in Figure 3 and the rapid changes in 
correlation dimensions that can be observed in general noise signals could not be observed. Therefore, it can be seen that the time series data used in this paper strongly show chaotic characteristics rather than the characteristics of noises.

\section{2-3. Qualitative analysis}

A representative method of qualitative analyses used to analyze the chaotic characteristics of time series signals is the power spectrum analysis method that analyzes chaotic characteristics through energy distributions on planes. When power spectrums are observed, it can be seen that periodic time series signals are concentrated on a certain frequency and pure noise signals show the energy distributed the entire sections of frequencies while the form of energy concentration of chaotic signals is in between that of periodic signals and that of noise signals and the energy is distributed in a relatively narrow area.[4] Figure 4 shows a power spectrum obtained from time series data and it can be seen that quite some characteristics of chaotic signals are shown as energy distributions are concentrated on a part of the areas.

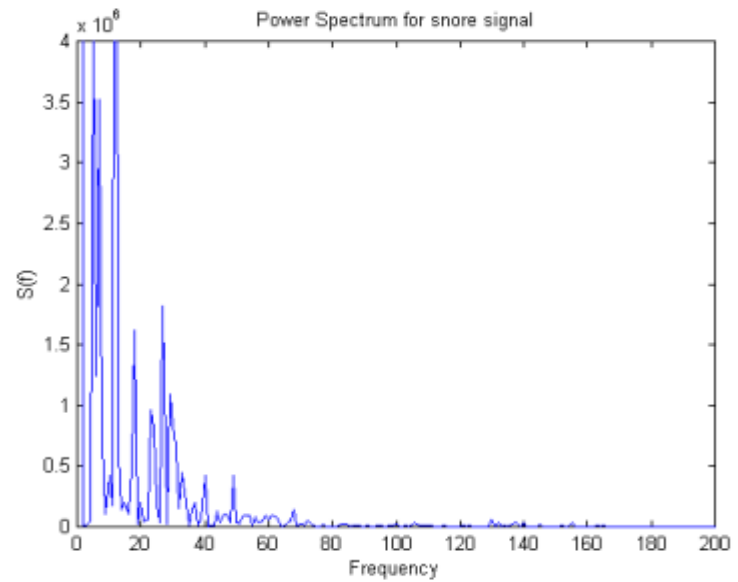

Fig. 4. Power spectrum of time series data

Auto-correlation functions are used as an analysis method to clearly distinguish between periodic signals and non-periodic signals. Although signals with periodicity are clearly identified, noise signals and chaotic signals are not clearly distinguished from each other when they are compared using auto-correlation functions thus it is difficult to distinguish between different signals.

Figure 5 shows the auto-correlation functions of time series data and it was identified that, although it is difficult to clearly distinguish them from noises, the average value of the auto-correlation functions was higher than that of noises.

\section{2-4. Judgment of the chaotic characteristics of time series models}

To check the existence of chaotic characteristics in the obtained time series data, quantitative and qualitative analysis methods were used. Snoring signals etc can be detected using the characteristics of time series data that intermittently show periodicity in case they have chaotic characteristics.[2][6] Through the quantitative and qualitative analyses mentioned earlier such the analysis of attracters in the phase space, correlation dimension and correlation integral analyses using embedding dimensions and power spectrum and auto-correlation function analyses, the obtained snoring signals were identified as chaotic signals that intermittently provide periodicity.

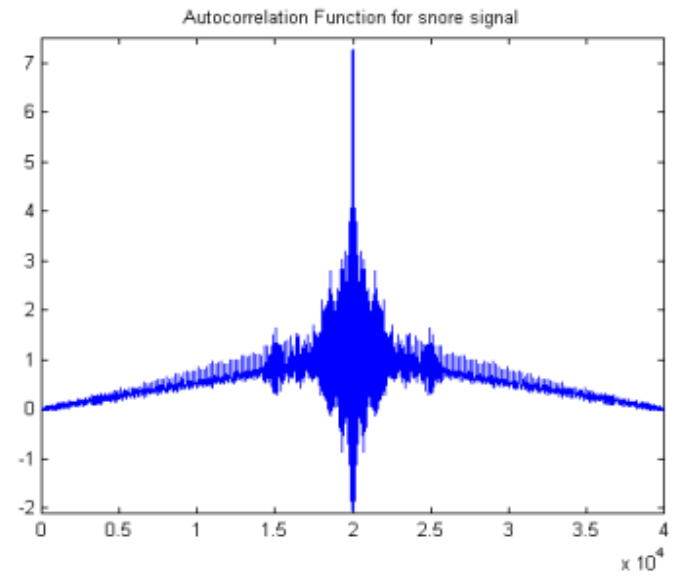

Fig. 5. Autocorrelation function of time series data

\section{SNORING DETECTION SYSTEM DESIGN}

\section{3-1. Things to be reviewed for the design}

In order to check the existence of chaotic characteristics in snoring signals before designing a snoring detection system, analyses were conducted with diverse methods such as quantitative methods and qualitative methods to grasp the characteristics of the time series data that intermittently provide periodicity.

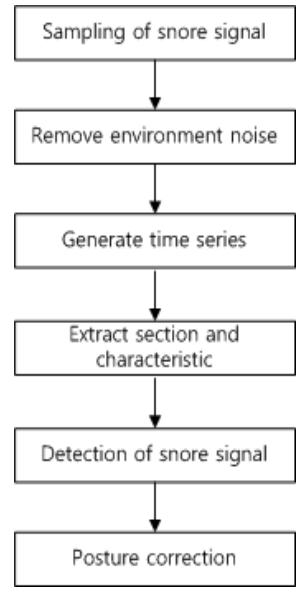

Fig. 6. Basic snoring signal detection flow chart 
To enhance the accuracy and reliability of the detection of snoring signals, the detection system should be designed considering the genders, signal sizes and sleeping times of diverse snoring patients. In this study, these considerations were reflected in the stage of designing the hardware due to the characteristics of the system that react at real times thereby enhancing the accuracy and reliability of snoring signal detection.

Figure 6 shows the basic operation processes of the snoring signal detection system as a flow chart. Inputted snoring signals go through a noise removing circuit and then they are converted into time series data. The converted data are judged whether they are similar to snoring signals through section and characteristic extraction processes.

\section{3-2. Detection algorithm}

Generally, an adult's respiratory rate is 12 to 20 times per minute in the air containing $20 \%$ oxygen and furthermore, respirations in sleeping are stabilized and have regularity. Although not the same over all sections, there are beginnings and ends of snoring signals that appear in periodic and similar forms.
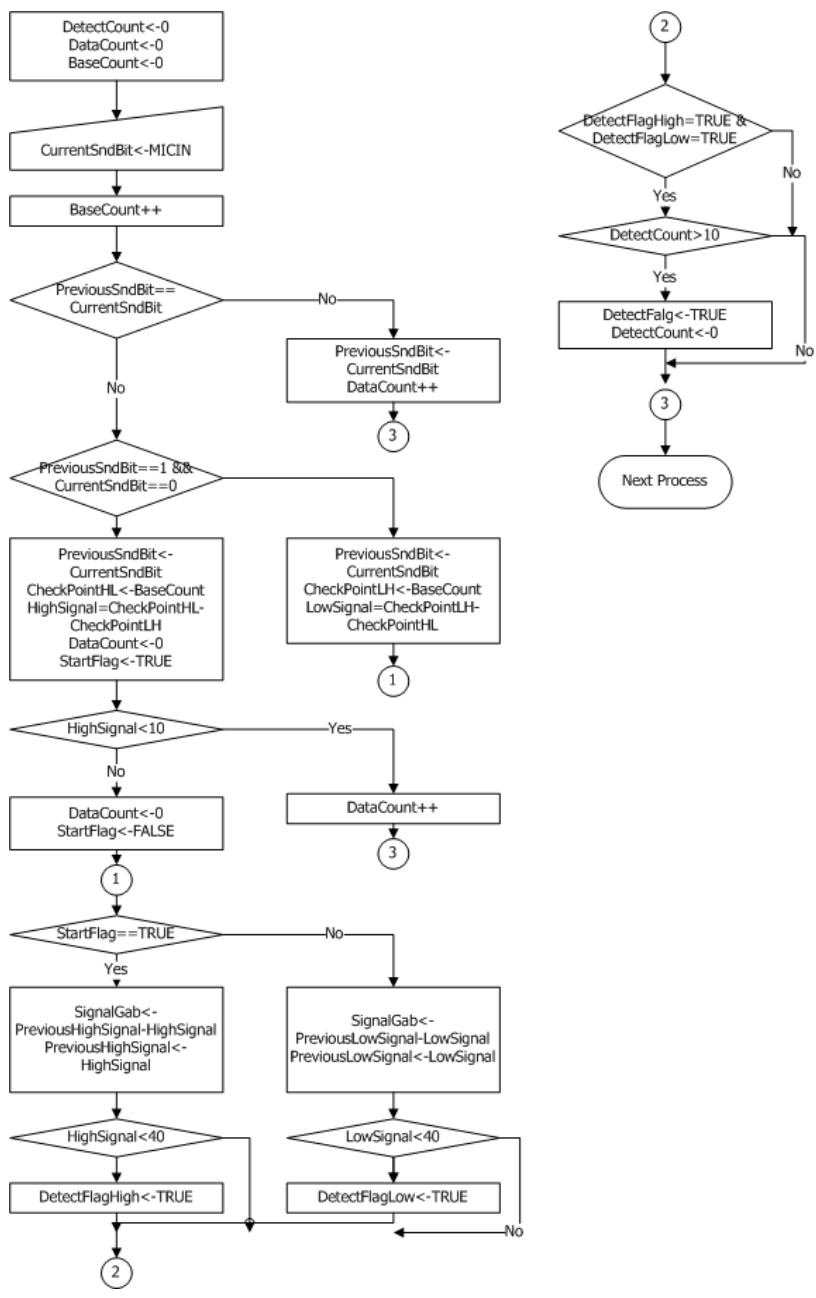

Fig. 7. Detection algorithm(part)
As identified through the analysis of the chaotic characteristics of snoring time series data, it was identified that snoring signals had periodicity in some sections. The system was designed to set the time during which snoring is maintained and the time for snoring to be repeated in these sections as major parameters to compare the size and periodicity of the signals successively occurred following the signals occurred earlier in order to judge whether the signals are typical snoring signals thereby detecting snoring signals. Figure 7 shows a part of the detection algorithm as a flow chart.

\section{EXPERIMENT AND DISCUSSION}

The hardware produced to check the performance of the proposed snoring signal detection system is largely divided into a display part to display the states, a control part to perceive snoring, a rechargeable battery to supply the power, a vibration motor driving part used to correct the posture of the snoring patient when snoring signals are detected.

Figure 8 shows the internal circuit of the snoring perception system and the left side is a display part and the right side is a control part. The display part is composed of a mike for voice signals, an FND to display alarm times, an input switch for function selections, an LED for state displays and a JACK for charging. The control part is composed of a signal input part to process inputted voice signals, a micro controller part in charge of accurate snoring signal judgment, a power supply device part in charge of power supply to the rechargeable battery and an added function part to provide various kinds of added functions.

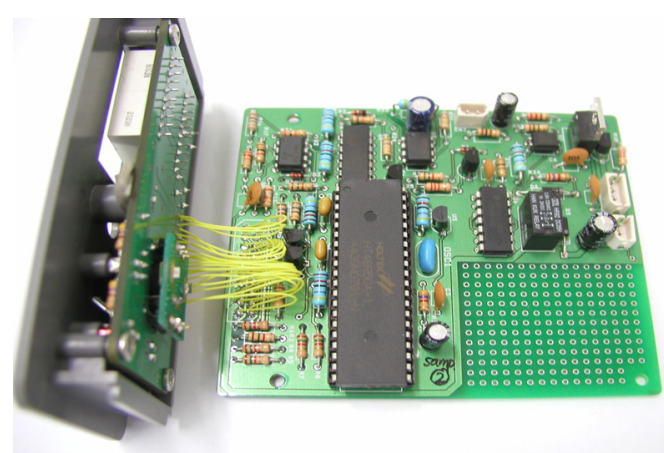

Fig. 8. The display part and the control part of the snoring detection system

The microcontroller applied to the snoring detection system is the HT48R50A1 which is a 8 bit high performance microcontroller of HOLTEK Co. This micro controller is compatible with the 8051 of Intel Co. and supports four 8 bit input/output ports and thus it enables snoring signal inputs and interfaces with diverse peripheral devices. Also, it was designed to implement the alarm function generally provided by RTC(Real-time 
Clock) by software using the timer/counter interrupt function of micro controllers in order to provide not only a snoring perception function but also related added functions. Figure 9 shows some of the peripheral circuits of the HT48R50A1 micro controller used in producing the hardware mentioned in this paper.

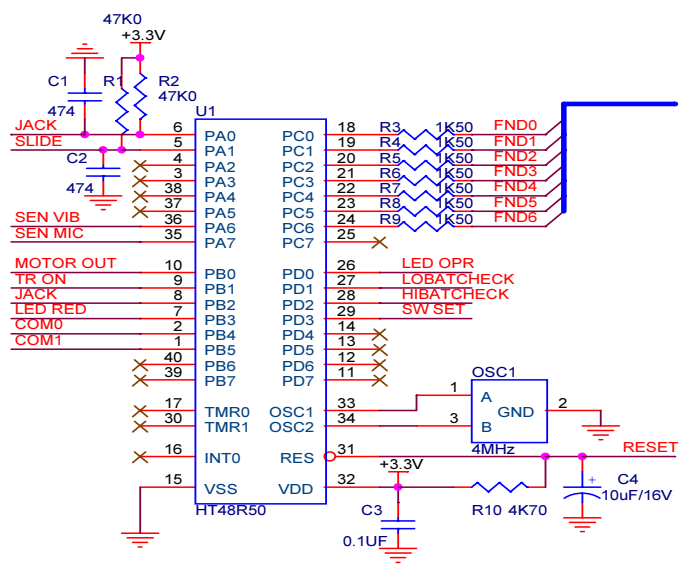

Fig. 9. Peripheral circuits of the micro controller

The hardware produced in this study was actually applied to a male snoring patient in his 30 s to identify the accuracy of the perception of the snoring detection system and the excellence of the applied algorithm. Figure 10 shows a waveform diagram obtained by an oscilloscope from the input/output ports of the micro controller using the voice signals obtained after inputting the snoring signals of the subject snoring patient to remove noises and the output signals processed by the perception algorithm. In the figure, the upper waveform shows logic signals made by filtering snoring voice signals and the lower waveform shows signals processed through the perception algorithm and these signals are diverse depending on snoring patients. To enhance the accuracy of detections, it is necessary to optimize the detection system by collecting signals of snoring patients composed of patients with diverse ages and different sexes.

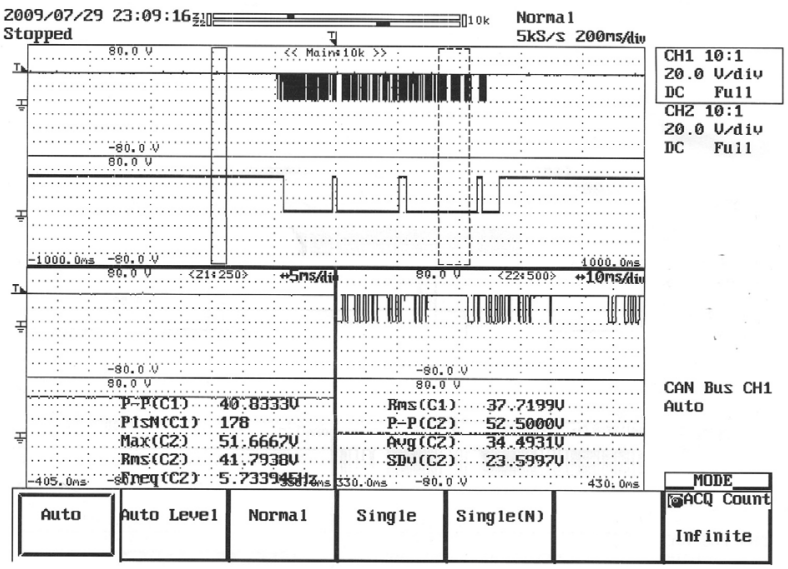

Fig. 10. Input signal and output signal of snoring
An experiment was conducted by applying a detection system applied with the algorithm designed in this study to a snoring patient. As a result, the system operated accurately on general voices existing in actual lives and even on environmental noises such as TV sounds. However, the detection rate differed depending on the direction of the face of the snoring patient when the patient was sleeping. This is due to the directivity of the mike that collects snoring voice signals and it is considered that the condition can be improved through improving the performance of the mike and the location of the installation of the mike.

In addition, to apply the proposed detection system to female snoring patients, some parameters related to sensitivity such as signal sizes, periodicity and breathing times should be reviewed as explained earlier.

\section{CONCLUSIONS}

In this study, the existence of chaotic characteristics in snoring signals obtained in the form of time series data was checked through quantitative and qualitative analysis methods, and a snoring signal detection system was designed applied with detection algorithms considering diverse parameters of occurring signals in order to enhance the accuracy and reliability of detections and the performance of the system was checked. The system was tested with certain snoring patients and thereby the results as follows could be obtained.

First, the detection algorithm considering the diverse parameters occurring from snoring signals was limited but satisfactory detection results could be obtained from snoring patients within the relevant range.

Second, the time of occurrence of snoring could be suppressed by imposing stimulating signals to correct the posture of the snoring patient when snoring was detected.

Third, the time of occurrence of snoring could be suppressed by imposing stimulating signals to correct the posture of the snoring patient when snoring was detected. It is considered that the snoring detection system designed to remove the physical factor of disorder of snoring patients can remove the adverse effects of surgeries and apparatuses for preventing snoring, induce changes in the perception of the risk and importance of snoring and finally, provide diverse advantages such as maintaining smooth home and social lives through the improvement sleeping environments. Although, areas to which snoring detection systems can be applied are limited now, it is judged that, through the development of modular products, the system can be applied to diverse areas such as beds, pillows for snoring prevention etc. It is also considered that the enhancement of the performance can be expected by complementing the design of the apparatus for effective posture corrections when snoring signals are detected and continuously collecting snoring signals of diverse age groups including females and applying them to analysis systems. 


\section{REFERENCES}

[1] T. Parker and L. Chua, "Chaos: A Tutorial for Engineers", Proc. of the IEEE, Vol.175, No.8, pp. 982-1008, 1987

[2] Heinz-otto Peitgen, Hartmut Jürgens, Dietmar Saupe, "Chaos and Fractals, New frontiers of science", Springer-Verlag, 1992

[3] Jong Hyun Kim and John Stringer, "Applied Chaos", A WileyInterscience Publication, 1992

[4] T. Shinbrot, C. Grebogi, E. Ott \& J. York, "Using Small perturbations to control chaos", Nature, Vol.1363, pp. 411-417, 1993

[5] W.Ditto and L.Pecora, "Mastering Chaos", Scientific American, Vol.67, No.8, p.62-68, 1993

[6] Edward Ott., "Chaos in Dynamical Systems", Cambridge University Press, 1993

[7] López, F. J., M. S., Behbehani, K. Ph. D., and Kamangar F., Ph.D. "An Artificial Neural Network Based Snore Detector", IEEE. pp. 1107-1108. 1994

[8] Steven H.Strogatz, "Nonlinear Dynamics and Chaos", Addison Wesley, Massachusetts, 1994

[9] G.L.Baker and J.P.Gollub, "Chaotic Dynamics", Cambridge University Press, New York, 1996

[10] T.H.Lee, U.R.abeyratne, "Analysis of Snoring Sounds for The Detection of Obstructive Sleep Apnea", Proceedings of the EMBEC, pp.538-539, 1999

[11] H. Y. Kim, H. J. Park, K. S. Park. "Real-Time Analysis of Snoring in Polysomnographic Recording". IEEE. pp.885. 1999

[12] Raimon Jané, Jordi Solà-Soler, José Antonio Fiz, Josep Morera. "Automatic Detection of Snoring Signals : Validation with Simple Snorers and OSAS Patients". Proceedings of the 22nd Annual EMBS. pp. 3129- 3131. 2000

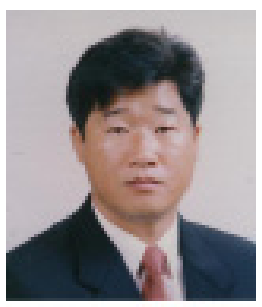

Yeon-Gyu Choo Received B.S. degree of Electronic Engineering in 1988 from Pukyong National University. and M.S. and Ph.D. degrees of Electronic Engineering in 1991 and 1997 respectively, from Dong-A University. In 1997, he joined the Jinju National University in Jinju, Korea, where is currently an associate professor and his research interests are in Intelligent Control System, Fuzzy Control System. 